\title{
DNA-methylated gene markers for colorectal cancer in TCGA database
}

\author{
HUI ZHANG, XUN SUN, YA LU, JIANZHONG WU and JIFENG FENG \\ Research Center for Clinical Oncology, The Affiliated Cancer Hospital of Nanjing Medical University, \\ Jiangsu Cancer Hospital, Jiangsu Institute of Cancer Research, Nanjing, Jiangsu 210000, P.R. China
}

Received June 4, 2019; Accepted November 21, 2019

DOI: $10.3892 / \mathrm{etm} .2020 .8565$

\begin{abstract}
Colorectal cancer (CRC) is characterized by the accumulation of genetic and epigenetic alterations in neoplastic processes. DNA methylation, as an important epigenetic process, contributes to the development of CRC. In the present study, the epigenetic landscape of genes in CRC was characterized by analyzing the dataset from The Cancer Genome Atlas database and 177 DNA-methylated genes were screened based on the criterion of the Pearson correlation (R) between expression and methylation levels being $>0.4$. Pathway enrichment analysis revealed prominent pathways, including transcription and metabolism, further implying their significant role in tumorigenesis. Among the methylated genes, only zinc finger protein (ZNF)726 with aberrant expression was determined to affect overall survival (OS) as well as disease-free survival of patients with CRC. In addition, ZNF726 was identified as an independent prognostic risk factor for OS in patients with $\mathrm{CRC}$. The methylation-based regulation of ZNF726 expression in CRC cells was further assessed using the Cancer Cell Line Encyclopedia database. Finally, the $\mathrm{CpG}$ island methylation of the ZNF726 promoter was evaluated to further elucidate its role in the development of CRC. In conclusion, the epigenetic landscape of genes in terms of promoter methylation in $\mathrm{CRC}$ was characterized, revealing that aberrant expression of ZNF726 may be an independent prognostic risk factor for OS in patients with CRC.
\end{abstract}

Correspondence to: Dr Jifeng Feng, Research Center for Clinical Oncology, The Affiliated Cancer Hospital of Nanjing Medical University, Jiangsu Cancer Hospital, Jiangsu Institute of Cancer Research, 42 Baiziting, Nanjing, Jiangsu 210000, P.R. China E-mail: jifeng_feng@163.com

Abbreviations: CRC, colorectal cancer; TCGA, The Cancer Genome Atlas; CCLE, Cancer Cell Line Encyclopedia; OS, overall survival; DFS, disease-free survival; ROC, receiver operating characteristic; AUC, area under the curve

Key words: DNA methylation, The Cancer Genome Atlas, gene expression, $\mathrm{CpG}$ island, zinc finger protein 726

\section{Introduction}

Colorectal cancer (CRC) is the third most commonly diagnosed cancer type worldwide. The incidence and death rates of CRC continue to decline in individuals aged $\geq 50$ years but continues to rise in those aged $<50$ years (1). In contrast to the survival rate of $\sim 65 \%$ for patients with localized disease, the 5-year survival rate of patients with metastatic CRC after diagnosis is $<10 \%$ (2). Hence, disease detection and treatment at an early stage are crucial (3). Considering the relatively high prevalence in the population, novel treatment strategies must be developed by characterizing the genetic, epigenetic, transcriptomic and proteomic changes to improve disease detection and surveillance. Epigenetic alterations have been established as a hallmark of tumorigenesis $(4,5)$. DNA methylation, as an important epigenetic process, is a common molecular alteration in CRC (6) and contributes to the later stages of colorectal carcinogenesis (7). Thus, DNA methylation of multiple promoters may serve as a biomarker for the early detection and monitoring of CRC progression. However, DNA methylation of genes and its consequences in $\mathrm{CRC}$ remain poorly characterized. In addition to global hypomethylation, genome-scale studies have yielded important insight into promoter methylation, including the $\mathrm{CpG}$-island methylator phenotype (CIMP), which displays extensive DNA hypermethylation at a unique set of $\mathrm{CpG}$ islands $(8,9)$. The Cancer Genome Atlas (TCGA) database (http://tcga-data. nci.nih.gov/tcga/) is a comprehensive molecular database of cancer genomes and epigenomes and has provided an excellent opportunity for analyses on molecular diagnostics for cancer $(10,11)$.

In the present study, methylation-regulated genes were investigated by integrating epigenetic and genomic data from the TCGA database. Pearson's correlation between gene methylation and expression was determined and the genes with $\mathrm{R}>0.4$ were regarded as methylated genes. In addition, methylation-regulated genes associated with the prognosis of patients with CRC (key genes) were identified by combining the data from the TCGA database. Furthermore, the key gene was validated in the Cancer Cell Line Encyclopedia (CCLE) database (https://portals.broadinstitute.org/ccle) (12). The $\mathrm{CpG}$ island methylation of key genes was further evaluated. The results demonstrated that zinc finger (ZNF)726, which was regulated by DNA methylation at the $\mathrm{CpG}$ islands in its 
promoter region, may be an independent prognostic risk factor for overall survival (OS) of patients with CRC.

\section{Materials and methods}

Screening of methylated genes in the TCGA database. The transcriptome profiling and DNA methylation data (IlIumina Human Methylation 450) were downloaded from the TCGA database. Finally, the data of 428 tissues (407 colorectal tumor tissues and 21 adjacent non-tumor tissues) were obtained, which contained RNA expression data and DNA methylation information. For gene methylation vs. expression, Pearson's correlation coefficients of $>0.4$ and $\mathrm{P}<0.05$ were considered to indicate methylation-regulated genes, and these were further studied. Patients without clinical information, including age, gender, TNM stage, residual tumor reaction margin and survival, were excluded. Finally, 390 patients with CRC were enrolled in the clinical relevance study.

Pathway analysis. Pathway enrichment analysis of DNA-methylated genes screened from TCGA was performed on the basis of the consensus PathDB database (http://cpdb. molgen.mpg.de/) (13). Over-representation analysis (14) was utilized for the DNA-methylated gene list. The following pathway databases were defined for the analysis: Manual upload, NetPath, Signalink, PID, EHMN, HumanCyc, INOH, KEGG, Biocarta, WikiPathways, SMPDB and PharmGKB (13). Minimum overlap input list $>2$ and $\mathrm{P}<0.05$ indicated statistical significance.

DNA-methylated genes predicting the survival of patients with $C R C$. To evaluate whether the expression of certain methylated genes was able to predict patient survival, 390 patients with CRC were divided into high and low expression groups based on the median of gene expression. The OS and disease-free survival (DFS) of patients in two subgroups for each gene were evaluated using Kaplan-Meier survival curves and the log-rank test. Prognosis-associated genes (key genes) were then obtained. It was inferred that if any of the key genes was an independent predictive factor for OS of CRC patients, the clinicopathologic features were analyzed using univariate and multivariable Cox proportional hazards models. The hazard ratio and $95 \%$ confidence interval (CI) were thereby determined. The diagnostic value of DNA-methylated genes in patients with CRC was further evaluated by using receiver operating characteristic (ROC) curve analysis. The area under the ROC curve (AUC) was subsequently calculated.

Experimental validation in the CCLE database. To compare the role of the ZNF726 gene in CRC with that in other cancer types, the expression and methylation levels of key genes were examined in 13 other types of tumor tissues from the TCGA database. Furthermore, 21 colorectal cancer cell lines from the CCLE database (https://portals.broadinstitute.org/ccle/about) were selected to detect the expression and methylation levels and their relevance was verified. The expression and methylation levels of key genes in tissues and cell lines were determined.

CpG island methylation of a key gene and its clinical value. To continue detecting the methylation levels of the promoter of the key gene, the $\mathrm{CpG}$ islands were downloaded and analyzed. The Pearson correlation between key gene expression and $\mathrm{CpG}$ island methylation was also evaluated. Finally, the methylation profile of $\mathrm{CpG}$ islands was further verified in Gene Expression Omnibus (GEO) datasets (https://www.ncbi.nlm.nih.gov/gds/).

Statistical analysis. The methylation and expression data from the TCGA were analyzed with R 3.5.1 software (https://www.r-project.org/) using MethylMix version 3.7 (15) and limma version 3.363 packages. The Kaplan-Meier survival analysis was performed using the survival package in R. Differences between the groups were assessed via Student's t-test and visualized by ggstatsplot (16) version 0.05 and ggplot2 version 3.0.0 (17) in R. The univariate/multivariate Cox proportional hazard regression analysis was performed using SPSS version 22 (IBM, Corp.). The association of global/CpG site methylation with the gene expression level was estimated by Pearson's correlation analysis. $\mathrm{P}<0.05$ was considered to indicate statistical significance.

\section{Results}

Screening of methylated genes in the TCGA database. To study the methylation regulation in CRC, transcriptome profiling and DNA methylation data were downloaded from the TCGA. A total of 428 samples (21 normal and 407 tumor tissues) containing expression and methylation data were included in the study. Subsequently, the Pearson correlation between the methylation and expression levels was assessed and genes with a correlation coefficient of $>0.4$ were regarded as methylated genes. A total of 177 methylation-regulated genes were thereby obtained. The expression and methylation levels and Pearson correlations were calculated and presented in Fig. 1 and Table SI.

Pathway analysis. Pathway enrichment analysis was performed to explore the possible mechanism of methylation-regulated genes in CRC. Gene expression, genomic transcription pathway and RNA polymerase II transcription were prominent pathways. The top 20 pathways are provided in Fig. 2.

Methylation-regulated ZNF726 predicts the OS and DFS of patients with $C R C$. On the basis of the median value of differentially expressed genes, 390 patients with CRC were divided into high and low expression groups and the Kaplan-Meier survival curves were drawn. As a result, three key genes (NXPE4, REP15 and ZNF726), which were able to effectively distinguish the patients' prognosis, were screened. Patients with high expression of neurexophilin and PC-esterase domain family member 4 (NXPE4), RAB15 effector protein (REP15) and ZNF726 had a significantly improved prognosis regarding OS (Fig. 3A). However, only the expression of ZNF726 was able to predict the DFS of patients with CRC (Fig. 3B). The three genes were significantly less expressed and hypermethylated in tumor tissues (Fig. 3C and D). The significant correlation between methylation and expression levels presented in Fig. 4A and B further suggested their important role in tumors.

Subsequently, ROC curves were plotted to evaluate the diagnostic value of NXPE4, REP15 and ZNF726 


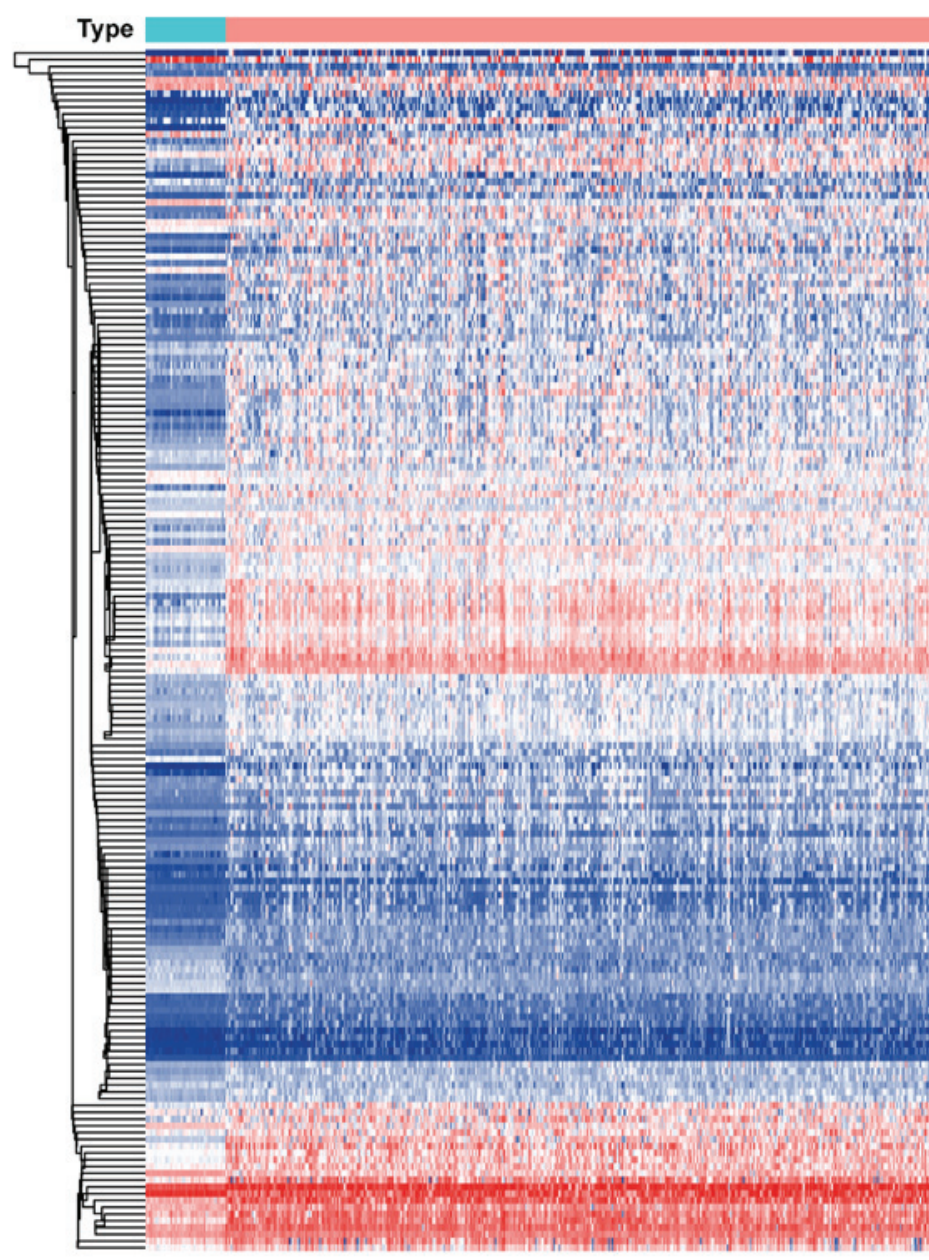

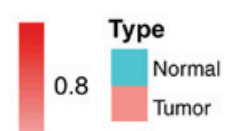

0.6

0.4

0.2

Figure 1. Heatmap of the DNA methylation profile between CRC tumor and non-tumor tissue in The Cancer Genome Atlas database. The full list of methylated genes is included in Table SI. CRC, colorectal cancer.

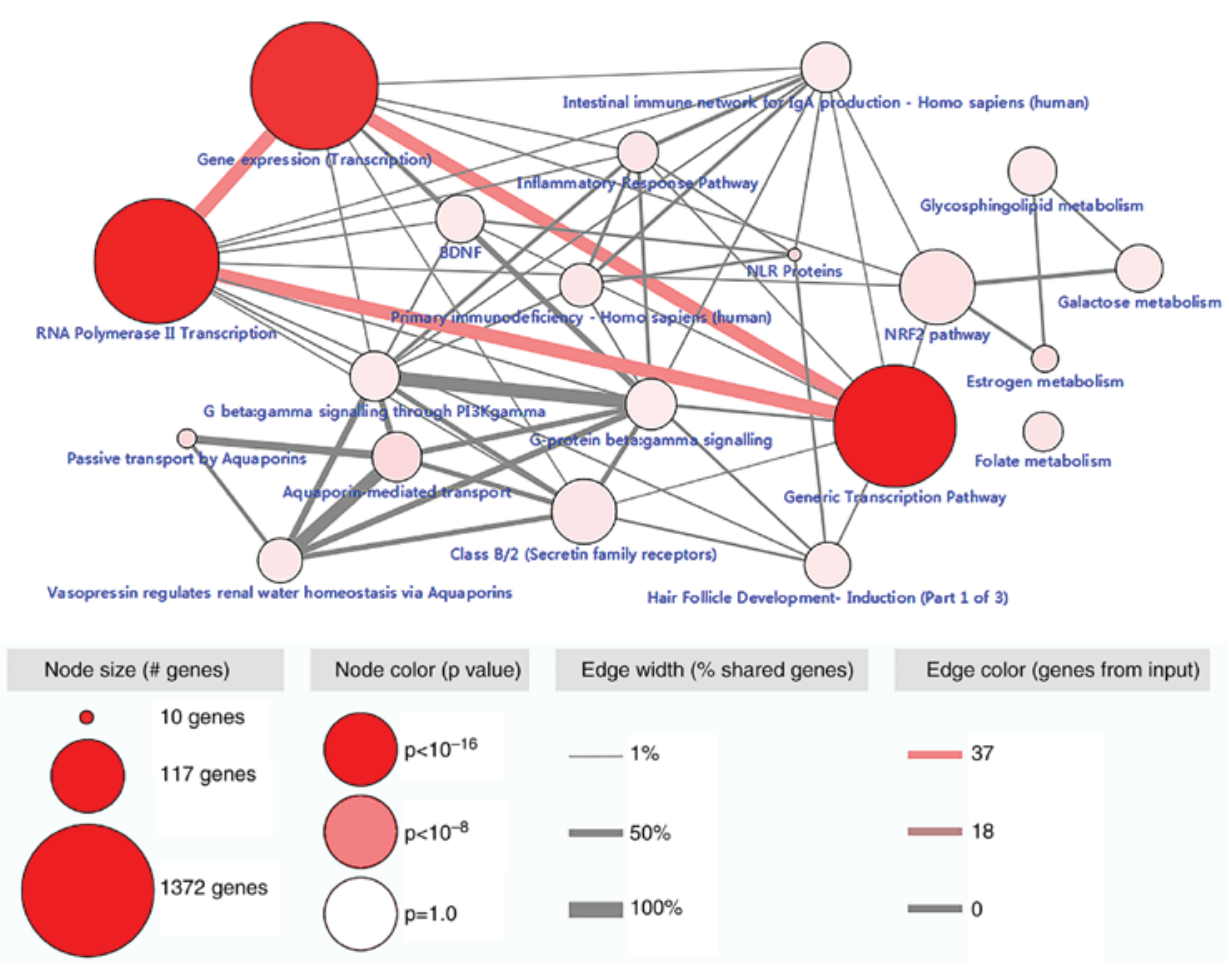

Figure 2. Pathways enriched by genes methylated based on MethylMix criteria. The analysis included all patients in The Cancer Genome Atlas cohort. $\mathrm{BDNF}$, brain-derived neurotrophic factor; NRF, nuclear respiratory factor. 
A

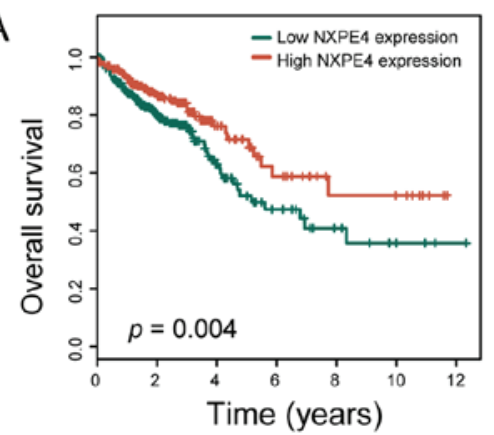

B

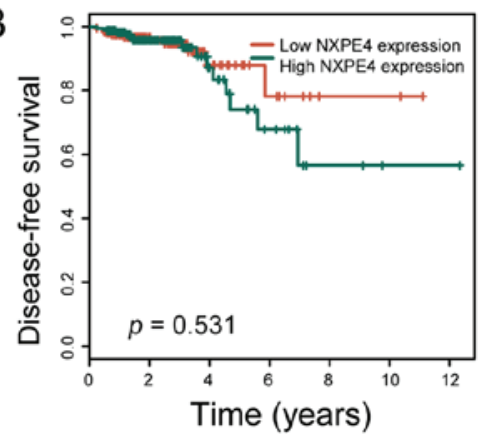

C
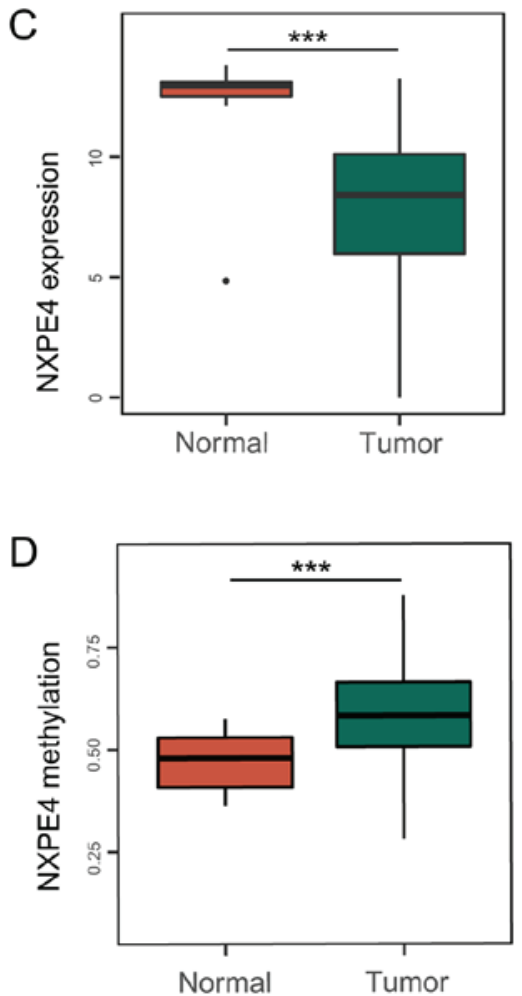
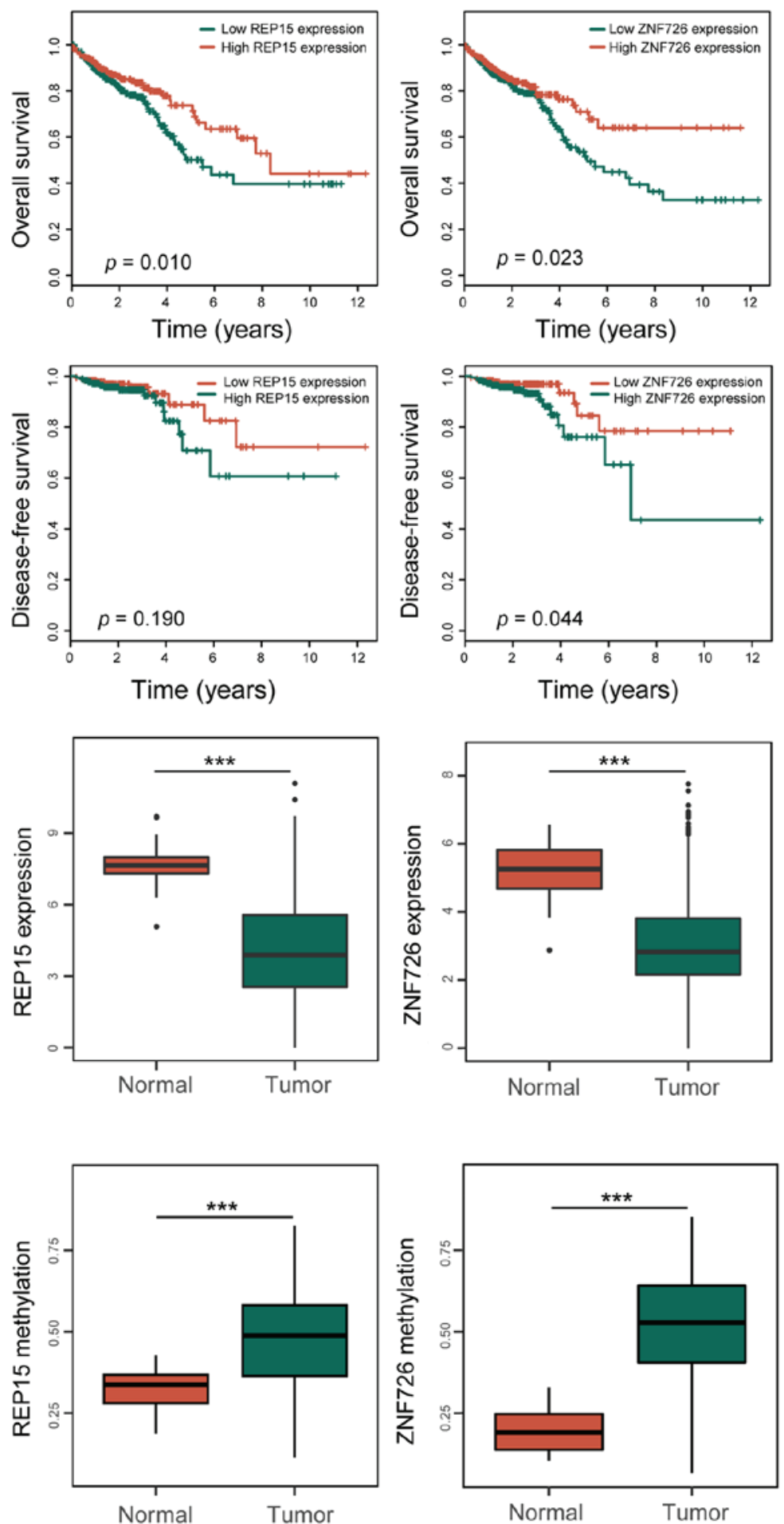

Figure 3. Methylation-regulated ZNF726 predicts the OS and DFS of patients with CRC. (A and B) Association between expression values of three candidate genes and CRC survival: (A) OS, (B) DFS, (C) Expression and (D) methylation levels of three genes between tumor and non-tumor tissues. ${ }^{* * *}$ P $<0.001$. OS, overall survival; DFS, disease-free survival.

methylation (Fig. 4C) between tumor and normal tissues. The AUCs for the three genes were 0.773, 0.792 and 0.929, respectively. The sensitivity and specificity were $(1.000,0.518)$, $(1.000,0.634)$ and $(1.000,0.860)$, respectively.

Cox regression analysis was performed to further verify whether ZNF726 may be used as an independent prognostic risk factor for $\mathrm{OS}$ in patients with $\mathrm{CRC}$. Univariate Cox analysis suggested that age $(\mathrm{P}<0.001)$, TNM stage $(\mathrm{P}<0.001)$, T stage
$(\mathrm{P}=0.003), \mathrm{N}$ stage $(\mathrm{P}<0.001), \mathrm{M}$ stage $(\mathrm{P}<0.001)$, residual tumor $(\mathrm{P}<0.001)$ and $\mathrm{ZNF} 726$ expression $(\mathrm{P}=0.024)$ were associated with prognosis and multivariate Cox regression analysis suggested that age $(\mathrm{P}=0.005)$, TNM stage $(\mathrm{P}<0.001)$, T stage $(\mathrm{P}=0.014), \mathrm{N}$ stage $(\mathrm{P}=0.036), \mathrm{M}$ stage $(\mathrm{P}=0.035)$, residual tumor $(\mathrm{P}=0.042)$, ZNF726 expression $(\mathrm{P}=0.015)$ were independent prognostic risk factors for OS in patients with CRC (Fig. 5). 

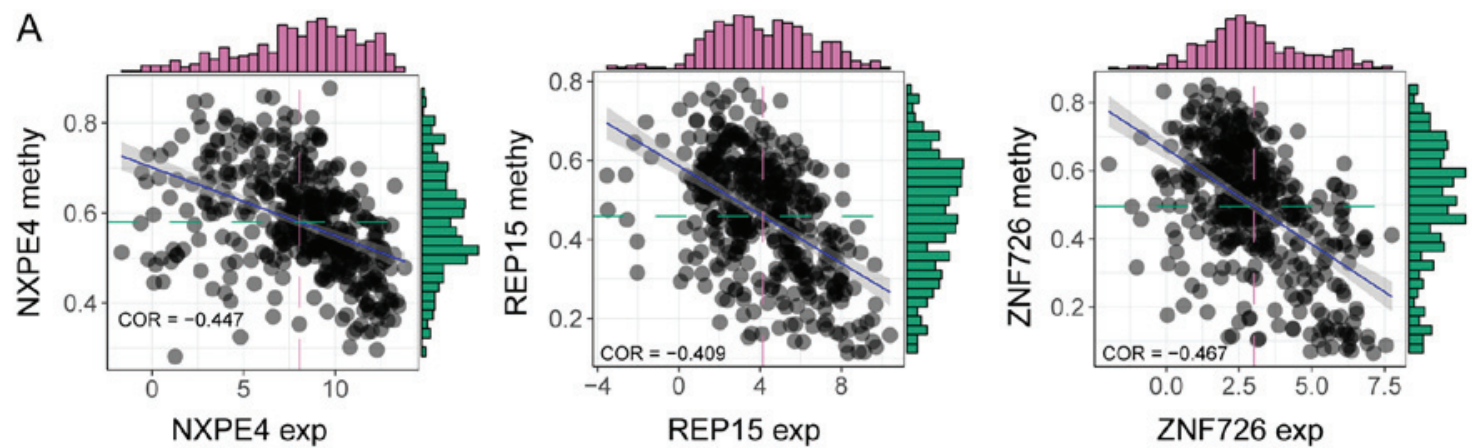

B
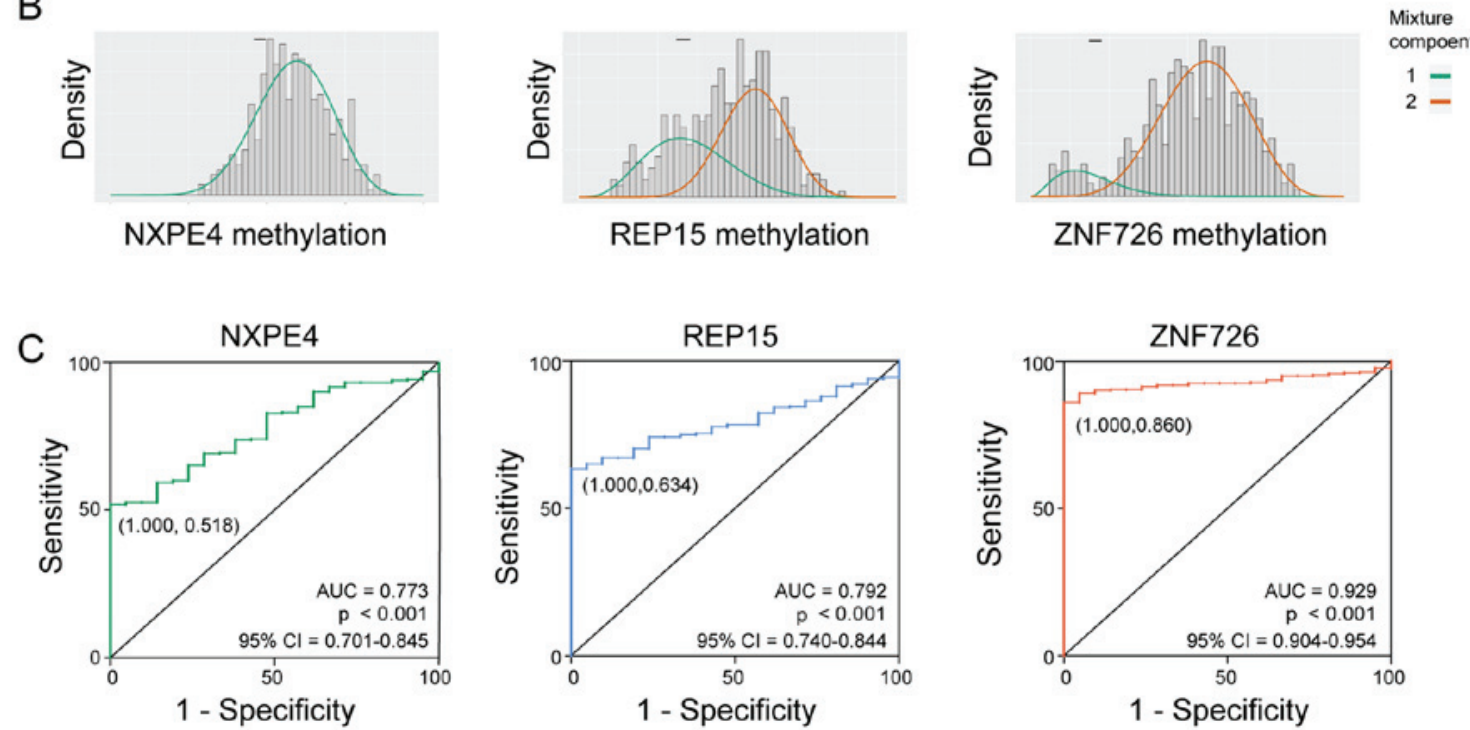

Figure 4. DNA methylation of three key genes, NXPE4, REP15 and ZNF726. (A) Pearson correlation between the expression and methylation of three prognosis-associated genes. (B) Summary of methylation of the genes. The histograms display the distribution of methylation among the tumor samples and the horizontal black bars represent the methylation values in the normal samples. The mixture components 1 and 2 represent the peak of methylation. (C) ROC curves of the three genes methylation for early diagnosis of patients with CRC from The Cancer Genome Atlas database. Exp, expression; methy, methylation; ROC, receiver operating characteristic; AUC, area under the ROC curve; R, Pearson correlation coefficient; REP15, RAB15 effector protein; ZNF, zinc finger protein; NXPE4, neurexophilin and PC-esterase domain family member 4.

Univariate analysis

Multivariate analysis

\begin{tabular}{|c|c|c|c|c|}
\hline & & & & \\
\hline Subgroup & Hazard ratio $(95 \% \mathrm{Cl})$ & P-value & Hazard ratio $(95 \% \mathrm{Cl})$ & P-value \\
\hline Age ( 265 vs. $<65$ years) & $1.973(1.327-2.934)$ & $<0.001$ & $2.120(1.251-3.592)$ & 0.005 \\
\hline Gender (Male vs. Female) & $1.102(0.769-1.579)$ & 0.597 & & \\
\hline TNM stage $(I I I+\mid \mathrm{V}$ vs. $|+|||)$ & $3.249(2.185-4.829)$ & $<0.001$ & $6.960(2.15-22.534)$ & $<0.001$ \\
\hline $\mathrm{T}$ stage $(\mathrm{T} 3+\mathrm{T} 4$ vs. $\mathrm{T} 1+\mathrm{T} 2)$ & $2.829(1.431-5.591)$ & 0.003 & $4.501(1.356-14.938)$ & 0.014 \\
\hline $\mathrm{N}$ stage (N1+N2 vs. N0) & $2.859(1.963-4.164)$ & $<0.001$ & $0.347(0.129-0.935)$ & 0.036 \\
\hline M stage (M1 vs. M0) & $4.4508(3.011-6.750)$ & $<0.001$ & $2.014(1.051-3.859)$ & 0.035 \\
\hline Residual tumor ( $R 1+R 2$ vs. $R 0)$ & 5.047 (3.047-8.359) & $<0.001$ & $1.915(1.023-3.585)$ & 0.042 \\
\hline $\begin{array}{l}\text { ZNF726 expression } \\
\text { (High vs. low) }\end{array}$ & $0.657(0.456-0.947)$ & 0.024 & $0.553(0.342-0.893)$ & 0.015 \\
\hline
\end{tabular}

Figure 5. Univariate and multivariate Cox regression analyses in patients with colorectal cancer. ZNF, zinc finger protein; yr, years.

Experimental validation in cancer tissues and CRC cell lines. The expression and methylation of ZNF726 were further examined in various types of tumor tissue. As presented in Fig. 6A and B, among 13 tumor tissue types, ZNF726 had the lowest expression and highest methylation in CRC, indicating that the role of ZNF726 in CRC may be linked to methylation regulation. Similarly, the expression and methylation levels of ZNF726 were negatively correlated in 21 CRC cell lines (Fig. 6C and D). These results further confirmed that ZNF726 was downregulated in CRC and regulated by DNA methylation. 

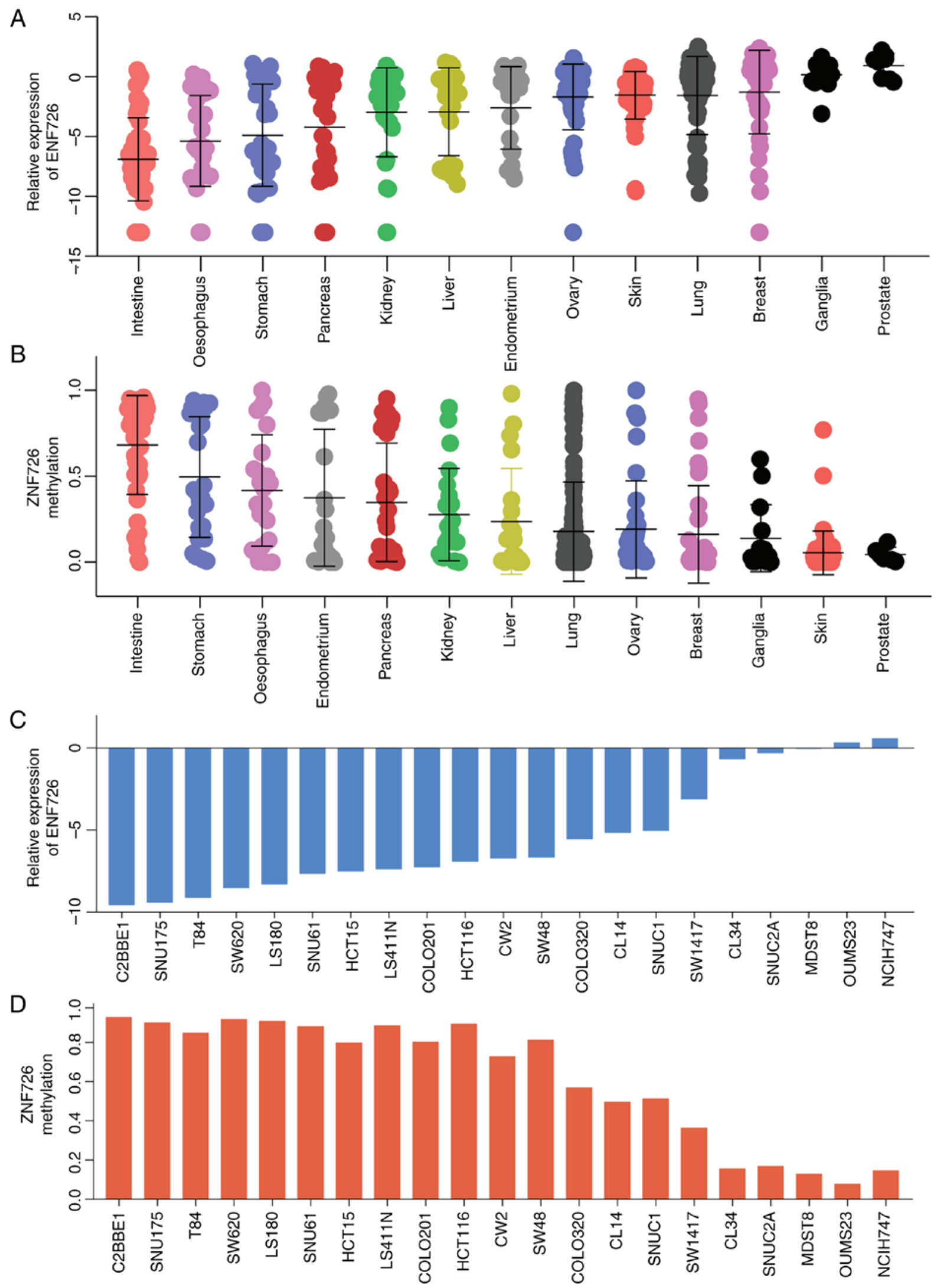

Figure 6. Verification of DNA-methylated ZNF726 in other tumor tissues and colorectal cell lines. (A) Relative expression and (B) methylation of ZNF726 in various tissues. (C) Relative expression and (D) methylation of ZNF726 in colorectal cancer cell lines. ZNF, zinc finger protein.

$C p G$ island methylation analysis of ZNF726. To further determine the mode of regulation of gene expression via methylation of the gene promoter, the $\mathrm{CpG}$ island methylation dataset was downloaded from the TCGA database. Only one $\mathrm{CpG}$ island (cg20649823) in the ZNF726 promoter was obtained. As presented in Fig. 7A, the methylation level of cg20649823 was significantly 
A

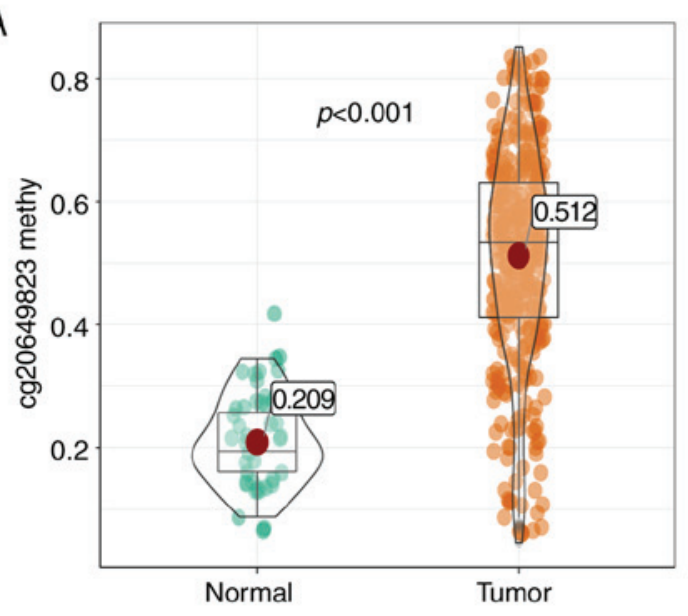

B

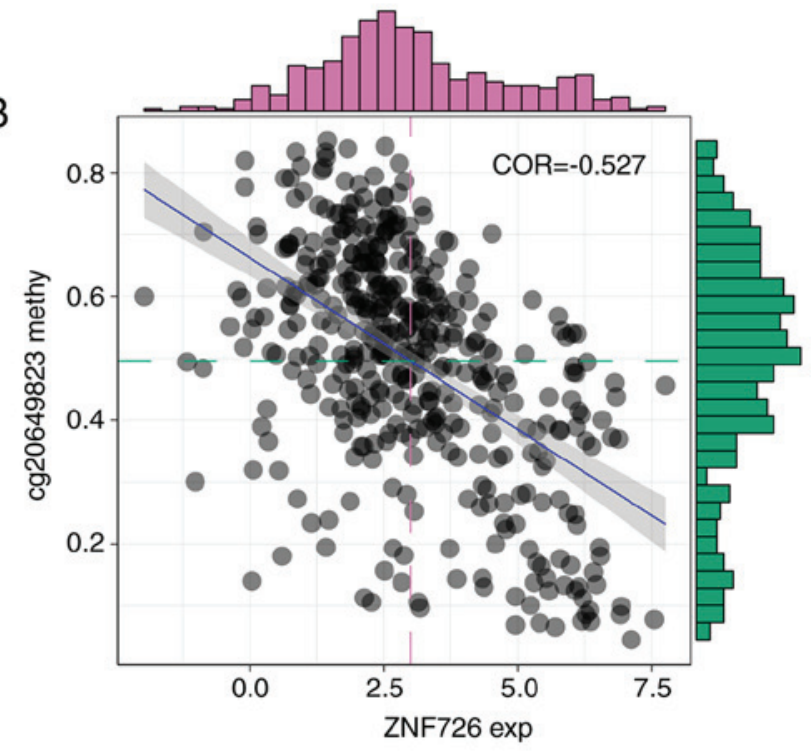

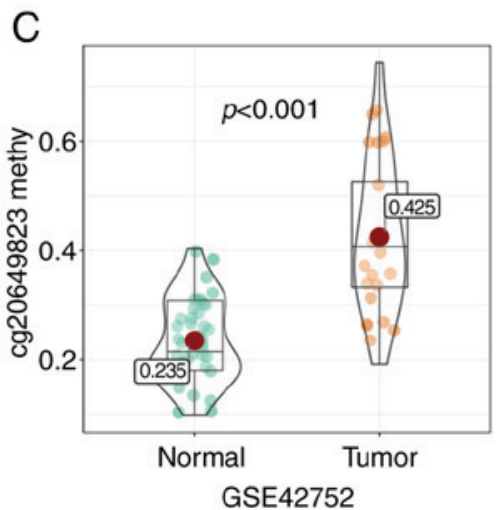
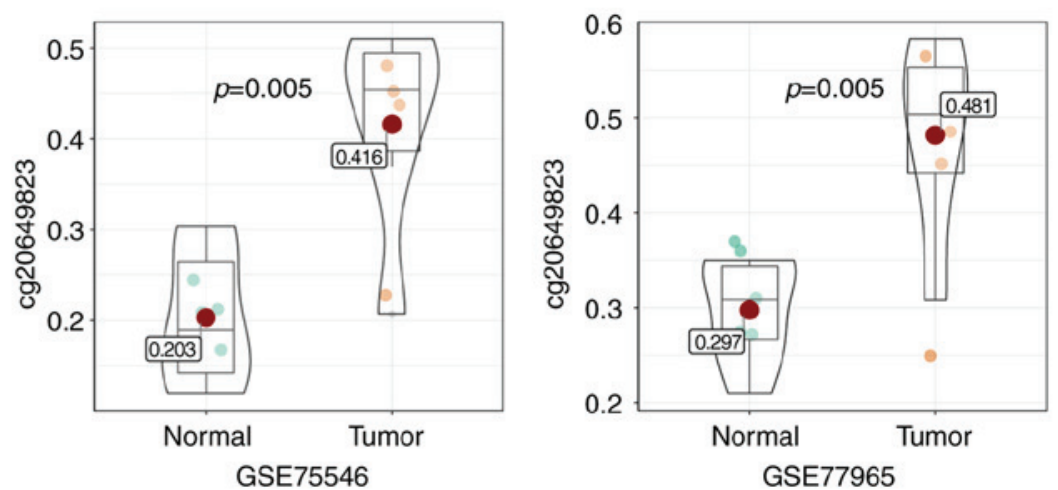

Figure 7. CpG-island methylation of ZNF726 promoter in The Cancer Genome Atlas database and its validation in the Gene Expression Omnibus database. The cg20649823 methylation between CRC tumor and non-tumor tissue in TCGA database (A) and in the GEO database (C); (B) Pearson correlation between ZNF726 expression and cg20649823 methylation. Exp, expression; methy, methylation; R, Pearson correlation coefficient; ZNF, zinc finger protein.

different between tumor and normal tissues. Furthermore, cg20649823 methylation and ZNF726 expression exhibited a significant correlation ( $\mathrm{R}=-0.527$; Fig. 7B). The methylation level of cg20649823 was also assessed in datasets from the GEO database (GSE42752, GSE75546 and GSE77965). As presented in Fig. 7C, based on the methylation level of cg20649823, it was possible to clearly distinguish CRC from normal tissues.

\section{Discussion}

CRC is characterized by the accumulation of genetic and epigenetic alterations in colonic epithelial cells in neoplastic processes. Cancer-specific genetic and epigenetic aberrations may be detected for non-invasive cancer diagnosis and monitoring (18). Aberrant hypermethylation of DNA at CpG islands, which mediates the transcriptional silencing of tumor suppressor genes, is an early event integral to gastrointestinal cancer development (3). Studies on the genetic and epigenetic modifications of molecular markers in human cancers in the TCGA database have opened new avenues for cancer detection approaches.

In the present study, CRC genomic and epigenetic data from the TCGA database were integrated to characterize the
DNA methylation landscape in CRC. It was inferred that the methylated genes targeted by epigenetic alterations in tumors may have an important role in tumor initiation and progression. Pathway enrichment analysis revealed prominent pathways, including those of transcription and metabolism, in tumorigenesis. Numerous known cancer-associated genes were included in the methylated gene set of the present study, certain of which are known to cause tumor development. For instance, the ectopic expression of transforming growth factor beta induced is associated with enhanced metastasis and extravasation of CRC (19-21). Polymeric immunoglobulin receptor promotes tumor growth in hepatocellular carcinoma (22), but inhibits pancreatic and periampullary adenocarcinoma development (23). Homeobox (HOX) expression contributes to CRC development: HOXB3, HOXB8 and HOXB9 are significantly upregulated, whereas HOXB2 and HOXB13 are significantly downregulated in carcinoma tissues (24). Furthermore, the aberrant regulation of bone marrow stromal cell antigen 2 is associated with breast cancer (25), hepatic carcinoma (26), esophageal cancer, gastric cancer and CRC $(27,28)$. The epigenetic regulation of E74-like ETS transcription factor 5 is associated with the metastasis of breast cancer (29) and urothelial cancer (30). 
Considering the clinical significance of methylation regulatory genes, Kaplan-Meier survival analysis was performed and the candidate key genes (NXPE4, REP15 and ZNF726), whose aberrant expression was linked to CRC prognosis, screened. ZNF proteins, including ZNF346, ZNF638, ZNF700 and ZNF768, are capture antigens for detecting autoantibodies in CRC (31). In addition, DNA methylation biomarkers of the ZNF gene family, combined with other genes, may be useful in the clinical setting for head and neck squamous cell carcinoma (32). Consistent with previous studies, the current study identified $44 \mathrm{ZNF}$ genes (Table SI) that are prominently regulated by methylation, further indicating the significance of the ZNF family in CRC development. Detection in multiple cancer types indicated that ZNF726 was the least expressed and most methylated in CRC, implying the high relevance of its methylation and marked oncogenic potential. Two novel genes (NXPE4 and REP15) associated with CRC prognosis require further research.

Analysis of the consequences of CIMPs on gene expression suggested that only a small percentage $(7 \%)$ of gene expression was downregulated with high CIMP in tumors. However, this downregulation appears to be enriched for genes essential for the genesis of CRC (33). The epigenetic driver ZNF726 identified in the present study featured CIMP DNA hypermethylation and reduced gene expression. The epigenetic driver genes are attractive therapeutic targets, considering that their activation by treatment with DNA methylation inhibitors may result in CRC occurrence. However, the present study lacks further clinical validation. In a follow-up study, the consistency of the level of ZNF726 in peripheral blood and $\mathrm{CRC}$ tissue will be further evaluated.

In conclusion, the present study presented a detailed knowledge base on the genes with altered DNA methylation and its associated pathways in CRC, paving the way for the development of novel cancer therapies. Epigenetically activated ZNF726 was identified as a novel prognosis-associated gene and may become a novel target for CRC treatment.

\section{Acknowledgements}

Not applicable.

\section{Funding}

This study was supported by grants from the Jiangsu Provincial Key Research Development Program (grant no. BE2016794 to JF and grant no. BE2016795 to JW).

\section{Availability of data and materials}

All data and materials are available from the TCGA (http:// tcga-data.nci.nih.gov/tcga/) and GEO databases https://www. ncbi.nlm.nih.gov/gds/).

\section{Authors' contributions}

$\mathrm{HZ}$ contributed to statistical analysis and manuscript preparation. XS and YL contributed to manuscript editing and the interpretation of data. JW and JF contributed to the design of the current study. All authors read and approved the final version of the manuscript.

\section{Ethics approval and consent to participate}

Not applicable.

\section{Patient consent for publication}

Not applicable.

\section{Competing interests}

The authors declare that they have no competing interests.

\section{References}

1. Siegel RL, Miller KD, Fedewa SA, Ahnen DJ, Meester RGS, Barzi A and Jemal A: Colorectal cancer statistics, 2017. CA Cancer J Clin 67: 177-193, 2017.

2. Weisenberger DJ, Liang G and Lenz HJ: DNA methylation aberrancies delineate clinically distinct subsets of colorectal cancer and provide novel targets for epigenetic therapies. Oncogene 37: 566-577, 2018.

3. Wong CC, Li W, Chan B and Yu J: Epigenomic biomarkers for prognostication and diagnosis of gastrointestinal cancers. Semin Cancer Biol 55: 90-105, 2019.

4. Jones PA and Baylin SB: The fundamental role of epigenetic events in cancer. Nat Rev Genet 3: 415-428, 2002.

5. Du Z, Fei T, Verhaak RG, Su Z, Zhang Y, Brown M, Chen Y and Liu XS: Integrative genomic analyses reveal clinically relevant long non-coding RNAs in human cancer. Nat Struct Mol Biol 20: 908-913, 2013

6. Tse JWT, Jenkins LJ, Chionh F and Mariadason JM: Aberrant DNA methylation in colorectal cancer: What should we target? Trends Cancer 3: 698-712, 2017.

7. Kim MS, Lee J and Sidransky D: DNA methylation markers in colorectal cancer. Cancer Metastasis Rev 29: 181-206, 2010.

8. Wang Z, Yang B, Zhang M, Guo W, Wu Z, Wang Y, Jia L, Li S, Cancer Genome Atlas Research Network, Xie W and Yang D: lncRNA epigenetic landscape analysis identifies EPIC1 as an oncogenic lncRNA that interacts with MYC and promotes cell-cycle progression in cancer. Cancer Cell 33: 706-720.e9, 2018.

9. Toyota M, Ahuja N, Ohe-Toyota M, Herman JG, Baylin SB and Issa JP: CpG island methylator phenotype in colorectal cancer. Proc Natl Acad Sci USA 96: 8681-8686, 1999.

10. Cancer Genome Atlas Network: Comprehensive molecular characterization of human colon and rectal cancer. Nature 487: 330-337, 2012.

11. Cancer Genome Atlas Research Network: Comprehensive molecular characterization of gastric adenocarcinoma. Nature 513: 202-209, 2014

12. Barretina J, Caponigro G, Stransky N, Venkatesan K, Margolin AA, Kim S, Wilson CJ, Lehár J, Kryukov GV, Sonkin D, et al: The cancer cell line encyclopedia enables predictive modelling of anticancer drug sensitivity. Nature 483: 603-607, 2012.

13. Kamburov A, Wierling $\mathrm{C}$, Lehrach $\mathrm{H}$ and Herwig $\mathrm{R}$ : ConsensusPathDB-a database for integrating human functional interaction networks. Nucleic Acids Res 37: D623-D628, 2009.

14. Sanford T, Meng MV, Railkar R, Agarwal PK and Porten SP: Integrative analysis of the epigenetic basis of muscle-invasive urothelial carcinoma. Clin Epigenetics 10: 19, 2018.

15. Gevaert O: MethylMix: An R package for identifying DNA methylation-driven genes. Bioinformatics 31: 1839-1841, 2015.

16. Indrajeet Patil: 'ggplot2' Based Plots with Statistical Details (R package ggstatsplot version 0.0.1). 2018 .

17. Wickham H: Ggplot2: Elegant Graphics for Data Analysis. J R Stat Soc 174: 245-246, 2011.

18. Xu RH, Wei W, Krawczyk M, Wang W, Luo H, Flagg K, Yi S, Shi W, Quan Q, Li K, et al: Circulating tumour DNA methylation markers for diagnosis and prognosis of hepatocellular carcinoma. Nat Mater 16: 1155-1161, 2017.

19. Ma C, Rong Y, Radiloff DR, Datto MB, Centeno B, Bao S, Cheng AW, Lin F, Jiang S, Yeatman TJ and Wang XF: Extracellular matrix protein betaig-h3/TGFBI promotes metastasis of colon cancer by enhancing cell extravasation. Genes Dev 22: 308-321, 2008. 
20. Yokobori T and Nishiyama M: TGF- $\beta$ signaling in gastrointestinal cancers: Progress in basic and clinical research. J Clin Med 6: E11, 2017.

21. Zhang H, Dong S and Feng J: Epigenetic profiling and mRNA expression reveal candidate genes as biomarkers for colorectal cancer. J Cell Biochem 120: 10767-10776, 2019.

22. Yue X, Ai J, Xu Y, Chen Y, Huang M, Yang X, Hu B, Zhang $\mathrm{H}$, He C, Yang X, et al: Polymeric immunoglobulin receptor promotes tumor growth in hepatocellular carcinoma. Hepatology 65: 1948-1962, 2017.

23. Richard Fristedt, Jacob Elebro, Alexander Gaber, Björn Nodin, Mathias Uhlén and Karin Jirström: Abstract B30: High expression of PIGR is an independent favorable prognostic factor in pancreatic and periampullary adenocarcinoma. Cancer Res 75: B30-B30, 2015.

24. Bhatlekar S, Fields JZ and Boman BM: HOX genes and their role in the development of human cancers. J Mol Med (Berl) 92: 811-823, 2014

25. Cai D, Cao J, Li Z, Zheng X, Yao Y, Li W and Yuan Z: Up-regulation of bone marrow stromal protein 2 (BST2) in breast cancer with bone metastasis. BMC Cancer 9: 102, 2009.

26. Pan XB, Qu XW, Jiang D, Zhao XL, Han JC and Wei L: BST2/Tetherin inhibits hepatitis $\mathrm{C}$ virus production in human hepatoma cells. Antiviral Res 98: 54-60, 2013.

27. Chiang SF, Kan CY, Hsiao YC, Tang R, Hsieh LL, Chiang JM, Tsai WS, Yeh CY, Hsieh PS, Liang Y, et al: Bone marrow stromal antigen 2 is a novel plasma biomarker and prognosticator for colorectal carcinoma: A secretome-based verification study. Dis Markers 2015: 874054, 2015

28. Mukai S, Oue N, Oshima T, Mukai R, Tatsumoto Y, Sakamoto N, Sentani K, Tanabe K, Egi H, Hinoi T, et al: Overexpression of transmembrane protein BST2 is associated with poor survival of patients with esophageal, gastric, or colorectal cancer. Ann Surg Oncol 24: 594-602, 2017.
29. Gallego-Ortega D, Ledger A, Roden DL, Law AM, Magenau A, Kikhtyak Z, Cho C, Allerdice SL, Lee HJ, Valdes-Mora F, et al: ELF5 drives lung metastasis in luminal breast cancer through recruitment of $\mathrm{Gr}^{+} \mathrm{CD}_{11 \mathrm{~b}^{+}}$myeloid-derived suppressor cells. PLoS Biol 13: e1002330, 2015.

30. Yao B, Zhao J, Li Y, Li H, Hu Z, Pan P, Zhang Y, Du E, Liu R and Xu Y: Elf5 inhibits TGF- $\beta$-driven epithelial-mesenchymal transition in prostate cancer by repressing SMAD3 activation. Prostate 75: 872-882, 2015.

31. O'Reilly JA, Fitzgerald J, Fitzgerald S, Kenny D, Kay EW, O'Kennedy R and Kijanka GS: Diagnostic potential of zinc finger protein-specific autoantibodies and associated linear B-cell epitopes in colorectal cancer. PLoS One 10: e0123469, 2015.

32. Gaykalova DA, Vatapalli R, Wei Y, Tsai HL, Wang H, Zhang C, Hennessey PT, Guo T, Tan M, Li R, et al: Outlier analysis defines zinc finger gene family DNA methylation in tumors and saliva of head and neck cancer patients. PLoS One 10: e0142148, 2015.

33. Hinoue T, Weisenberger DJ, Lange CP, Shen H, Byun HM, Van Den Berg D, Malik S, Pan F, Noushmehr H, van Dijk CM, et al: Genome-scale analysis of aberrant DNA methylation in colorectal cancer. Genome Res 22: 271-282, 2012.

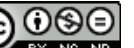

This work is licensed under a Creative Commons Attribution-NonCommercial-NoDerivatives 4.0 International (CC BY-NC-ND 4.0) License. 